\title{
Homotopy Perturbation Analysis in an energy transit problem closed to a Stretching Surface
}

\author{
Manoj Kumar Sarma ${ }^{1}$ and Sujan Sinha ${ }^{2}$ \\ ${ }^{1}$ Research Scholar, Department of Mathematics, Assam downtown University, Guwahati, India \\ ${ }^{2}$ Assistant Professor, Department of Mathematics, Assam downtown University, Guwahati, India \\ 1Email: mksghy3009@gmail.com²,Email: mathssujangu@gmail.com
}

Article History:Received:11 november 2020; Accepted: 27 December 2020; Published online: 05 April 2021

ABSTRACT : Present study explored the influence of stretching constraint in addition with inclusion of energy. An analytical solution for the system of non-linear equations of motion is worked out by adopting Homotopy Perturbation method (HPM). Physical and graphical reflections various parameters are demonstrated in the present problem. Utility of this model has been perceived in diverse industrial and chemical processes.

2010 AMS subject classification: 76 W05

Keywords: Homotopy Perturbation Method, Stretching Sheet, Stagnation point, porosity parameter

\section{Introduction}

Investigation of heat transfer in boundary layer has got tremendous application in Engineering, Plasma physics etc. and become an important subject for many researchers at the present time. In 1911, Hiemenz [9] considered the 2-D fluid flow near the point of stagnation and used similarity transformation. Thereafter, mass transfer, heat transfer, stagnation point flow, different physical effects, and stagnation point, calculation of skinfriction was exercised numerous ways.

The analytic form of 2-D steady flow of incompressible fluid because of the stretch sheet was estimated by Crane [5] with varying velocity. Also, Crane and Carragher [2] discussed the transfer of heat in above given flow model. Discussion of two-dimensional flow of stagnation point on the stretch surface of an incompressible viscous fluid was initiated by Chiam [4] and Mahapatra et al. [12] carried out the investigation by introducing temperature distribution. The pioneer work regarding the study of heat generation on a stretching surface was introduced in references [2], [7], [6], [13] and carried out their research by following the different phases of the problem.

The present research includes the experiment in laminar flow in a porous media when a viscous and incompressible fluid was used on a stretch surface with generation of heat considering the stream and wall temperatures to be constant. In this proposed study, the results of Attia [1], S. Kazem et al. [11] were modified by employing Homotopy Perturbation Method (HPM) He J. H. [8] to obtain almost exact solution of the energy and momentum governing equations. HPM is a constructive tool to covenant with nonlinear differential equation, has been established by many authors like A. K. Jhankal [10]. Though a huge calculation is involved in this analytic method (HPM), but always provides a convenient solution to discuss the behavior of the parameters. We sketched and discussed the obtained results for discussing the heat and flow characteristics. In addition to this, the outcomes were compared with those of Attia [1] and S. Kazem et al. [11] to verify the accuracy of our result. Moreover, we have shown and discussed one comparison graph related to temperature with the graph obtained by Attia [1], to establish the accuracy of the method adopted here.

\section{Mathematical formulation of the problem:}

A 2-dimensional steady stagnation flow of point for an incompressible and viscous, electrically conducting fluid near $\mathrm{O}$ the stagnation point, on a surface which is considered along the plane $\mathrm{y}=0$ ( $\mathrm{x}$-axis). The substantial circumstances describing the model with initial velocity $\boldsymbol{u}_{w}, \boldsymbol{T}_{w}$ be the temperature whereas $u_{w}(x), T_{\infty}(x)$ are the flow velocity and temperature which are calculated externally for a boundary layer as given in Figure 1.

Figure 1: Physical model of the problem

Figure 1: Physical model of the problem

Figure 1: Physical model of the problem 


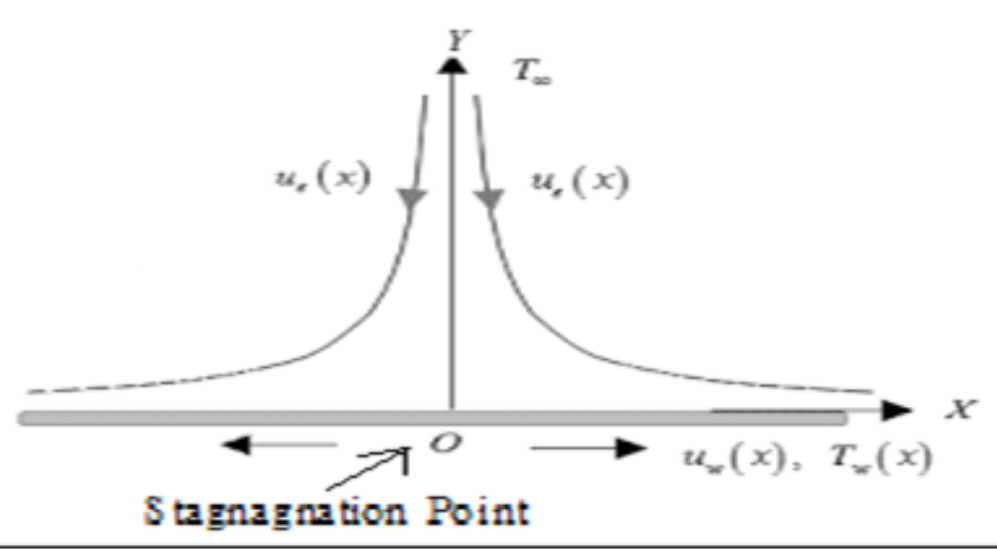

Figure 1: Physical model of the problem

Momentum equation, Continuity equation and the governing equation for two-dimensional steady flow, using standard boundary layer approximations Nazar et. al. [13], reduces "as follows:

$$
\begin{aligned}
& \frac{\partial u}{\partial x}+\frac{\partial v}{\partial y}=0 \\
& \rho\left(u \frac{\partial u}{\partial x}+v \frac{\partial u}{\partial y}\right)=U \frac{d U}{d x}+\mu \frac{\partial^{2} u}{\partial y^{2}}+\frac{\mu}{K}[U(x)-u]
\end{aligned}
$$

Where ' $\mathrm{K}$ ' is the Darcy Permeability; $\mu$ ' is coefficient of viscosity, \&' $\rho$ ' is fluid density of a fluid. Neglecting the dissipation, the governing energy equation with the temperature vales that depends on the absorption and generation of heat as per White M. F. [14],

$$
\rho C_{p}\left(u \frac{\partial T}{\partial x}+v \frac{\partial T}{\partial y}\right)=k \frac{\partial^{2} T}{\partial y^{2}}+Q\left(T-T_{\infty}\right)
$$

Where, the symbols have their usual meaning

The boundary limitations describing the flow are given by:

$$
\left.\begin{array}{l}
u=u_{w}(x)=c x, v=0 ; T=T_{w} \quad \text { for } y=0 \\
u=u_{e}(x) \rightarrow \alpha x ; \quad T \rightarrow T_{\infty} \quad \text { for } y \rightarrow \infty
\end{array}\right\}
$$

Here $\mathrm{c}$ is any positive constant, and $T_{w}$ is the temperature at the wall.

Using, $v=\mu / \rho$ as kinematic viscosity, using similarity transformations:

$$
u(x, y)=c x f^{\prime}(\eta), v(x, y)=-\sqrt{c v} f(\eta), \eta=\sqrt{\frac{c}{v}} y,
$$

Here the prime denotes the derivative value as per the value of $\eta$. For normalizing this model of flow, the non-dimensional temperature given below is introduced:

$$
\theta(\eta)=\frac{T-T_{\infty}}{T_{w}-T_{\infty}}, \operatorname{Pr}=\frac{\mu c_{p}}{K}, M=\frac{v}{c K}, C=\frac{\alpha}{c}, \quad B=\frac{Q}{c \rho C_{p}}
$$

The following nonlinear coupled differential equations are produced by applying (5), (6) in (2), (3) and

$$
\begin{aligned}
& f^{\prime \prime \prime}(\eta)+f(\eta) \cdot f^{\prime \prime}(\eta)-f^{\prime 2}(\eta)+M\left(C-f^{\prime}(\eta)\right)+C^{2}=0 \\
& \theta^{\prime \prime}(\eta)+\operatorname{Pr} . f(\eta) \cdot \theta^{\prime}(\eta)+\operatorname{Pr} \cdot B \cdot \theta=0 \\
& \left.\begin{array}{l}
f(\eta)=0, f^{\prime}(\eta)=1, \theta(\eta)=1 \text { as } \eta=0 \\
f^{\prime}(\eta) \rightarrow C, \theta(\eta) \rightarrow 0 \quad \text { as } \eta \rightarrow \infty
\end{array}\right\}
\end{aligned}
$$


$\operatorname{Pr}=\frac{\mu c_{p}}{K} \rightarrow$ stretching parameter, $M=\frac{v}{c K} \rightarrow$ porosity parameter,

$C=\frac{\alpha}{c} \rightarrow$ stretching parameter, $B=\frac{Q}{c \rho C_{p}} \rightarrow$ dimensionless heat generation / absorption coefficient

\section{Solution of the problem}

The nonlinear coupled differential equations (7) and (8) can be rewritten as:

$$
\begin{aligned}
& f^{\prime \prime \prime}+f \cdot f^{\prime \prime}-f^{\prime 2}-M \cdot f^{\prime}+M_{1}=0 \\
& \theta^{\prime \prime}+\operatorname{Pr} . f . \theta^{\prime}-M_{2} \theta=0
\end{aligned}
$$

Constants are mentioned in the appendix

Applying HPM, the equations (10), (11) can take the following form:

$$
\begin{aligned}
& (1-p)\left(f^{\prime \prime \prime}-M \cdot f^{\prime}\right)+p\left(f^{\prime \prime \prime}+f . f^{\prime \prime}-f^{\prime 2}-M \cdot f^{\prime}\right)=-M_{1} \\
& (1-p)\left(\theta^{\prime \prime}-M_{2} \theta\right)+p\left(\theta^{\prime \prime}+\operatorname{Pr} . f \cdot \theta^{\prime}-M_{2} \theta\right)=0
\end{aligned}
$$

We consider " $f$ " and " $\theta$ " are considered as under:

$$
\left.\begin{array}{l}
f=f_{0}+p f_{1}+p^{2} f_{2}+\ldots \ldots \ldots \ldots . \\
\theta=\theta_{0}+p \theta_{1}+p^{2} \theta_{2}+\ldots \ldots \ldots \ldots . .
\end{array}\right\}
$$

Using (14) in (12) and (13) and equating the terms free from 'p', the following ordinary differential equations are obtained:

$$
\begin{aligned}
& f_{0}^{\prime \prime \prime}-M \cdot f_{0}^{\prime}=-M_{1} \\
& \theta_{0}^{\prime \prime}-M_{2} \cdot \theta_{0}=0
\end{aligned}
$$

Solving these ordinary differential equations with the boundary conditions for zeroth order i.e.,

$$
\begin{array}{cc}
f_{0}(o)=0, f_{0}^{\prime}(0)=1, f_{0}^{\prime}(6)=C ; \theta_{0}(0)=1, \theta_{0}(6)=0 \text { we obtain the following solutions: } \\
f_{0}(\eta)=C_{1}+C_{2} e^{\sqrt{M} \eta}+C_{3} e^{-\sqrt{M} \eta}+\frac{M_{1}}{M} \eta & \rightarrow(17) \\
\theta_{0}(\eta)=C_{4} e^{\sqrt{M_{2}} \eta}+C_{5} e^{-\sqrt{M_{2}} \eta} & \rightarrow(18)
\end{array}
$$

Again, equating the terms involving coefficients of 'p' from the equation obtained by using (14) in (12) and (13), the following ordinary differential equations are obtained:

$$
\begin{aligned}
& f_{1}^{\prime \prime \prime}-M \cdot f_{1}^{\prime}+f_{0} \cdot f_{0}^{\prime \prime}-f_{0}^{2}=0 \\
& \theta_{1}^{\prime \prime}-M_{2} \theta_{1}+\operatorname{Pr} \cdot f_{0} \cdot \theta_{0}^{\prime}=0
\end{aligned}
$$

Solving these equations with the boundary conditions for $1^{\text {st }}$ order

i.e. $f_{1}(o)=0, f_{1}^{\prime}(0)=0, f_{1}^{\prime}(6)=0 ; \theta_{1}(0)=0, \theta_{1}(6)=0$ we obtain the following solutions:

$$
\begin{array}{r}
f_{1}(\eta)=C_{6}+C_{7} e^{\sqrt{M} \eta}+C_{8} e^{-\sqrt{M} \eta}+A_{10} \eta^{2}+A_{11} \eta^{3}+A_{12} \eta-\left[A_{15} e^{2 \sqrt{M} \eta}-A_{16} e^{-2 \sqrt{M} \eta}\right] \\
-\eta\left[E_{1} e^{\sqrt{M} \eta}+E_{2} e^{-\sqrt{M} \eta}\right]+\eta^{2}\left[A_{17} e^{\sqrt{M} \eta}+A_{18} e^{-\sqrt{M} \eta}\right] \\
\begin{array}{r}
\theta_{1}(\eta)=C_{9} e^{\sqrt{M_{2}} \eta}+C_{10} e^{-\sqrt{M_{2}} \eta}+\eta\left[A_{39} e^{\sqrt{M_{2}} \eta}+A_{40} e^{-\sqrt{M_{2}} \eta}\right]-\eta^{2}\left[A_{35} e^{\sqrt{M_{2}} \eta}-A_{36} e^{-\sqrt{M_{2}} \eta}\right] \\
-\left[A_{31} e^{M_{3} \eta}-A_{32} e^{-M_{3} \eta}\right]+\left[A_{33} e^{M_{4} \eta}-A_{34} e^{-M_{4} \eta}\right]
\end{array}
\end{array}
$$

Neglecting higher order perturbed terms we finally obtain: 


$$
\begin{aligned}
f(\eta)= & f_{0}+p f_{1} \\
= & C_{1}+C_{2} e^{\sqrt{M} \eta}+C_{3} e^{-\sqrt{M} \eta}+\frac{M_{1}}{M} \eta \\
& +p\left(\begin{array}{r}
C_{6}+C_{7} e^{\sqrt{M} \eta}+C_{8} e^{-\sqrt{M} \eta}+A_{10} \eta^{2}+A_{11} \eta^{3}+A_{12} \eta-\left[A_{15} e^{2 \sqrt{M} \eta}-A_{16} e^{-2 \sqrt{M} \eta}\right] \\
\left.-\eta\left[E_{1} e^{\sqrt{M} \eta}+E_{2} e^{-\sqrt{M} \eta}\right]+\eta^{2}\left[A_{17} e^{\sqrt{M} \eta}+A_{18} e^{-\sqrt{M} \eta}\right]\right)
\end{array}\right.
\end{aligned}
$$

And,

$$
\begin{aligned}
& \theta(\eta)=\theta_{0}+p \theta_{1} \\
& =C_{4} e^{\sqrt{M_{2}} \eta}+C_{5} e^{-\sqrt{M_{2}} \eta} \\
& +p\left(\begin{array}{rl}
C_{9} e^{\sqrt{M_{2}} \eta}+C_{10} e^{-\sqrt{M_{2}} \eta}+\eta\left[A_{39} e^{\sqrt{M_{2}} \eta}\right. & \left.+A_{40} e^{-\sqrt{M_{2}} \eta}\right]-\eta^{2}\left[A_{35} e^{\sqrt{M_{2}} \eta}-A_{36} e^{-\sqrt{M_{2}} \eta}\right] \\
& \left.-\left[A_{31} e^{M_{3} \eta}-A_{32} e^{-M_{3} \eta}\right]+\left[A_{33} e^{M_{4} \eta}-A_{34} e^{-M_{4} \eta}\right]\right)
\end{array}\right.
\end{aligned}
$$

The terminologies for viscous drag $(\tau)$ and Nusselt number $(\mathrm{Nu})$ are articulated as:

$$
\begin{gathered}
\tau=\left(\frac{\partial f}{\partial \eta}\right)_{\eta=0}=\sqrt{M}\left(C_{2}-C_{3}\right)+\frac{M_{1}}{M} \\
+p\left[\sqrt{M}\left(C_{7}-C_{8}\right)+A_{12}-2 \sqrt{M}\left(A_{15}+A_{16}\right)-\left(E_{1}+E_{2}\right)\right] \\
N u=-\left(\frac{\partial \theta}{\partial \eta}\right)_{\eta=0}=-\sqrt{M_{2}}\left(C_{4}-C_{5}\right)-p\left[\sqrt{M_{2}}\left(C_{9}-C_{10}\right)+A_{39}+A_{40}-M_{3}\left(A_{31}+A_{32}\right)+M_{4}\left(A_{33}+A_{34}\right]\right.
\end{gathered}
$$

\section{Findings}

The present analysis reveals the expressions of boundary layer equations with viscous drag and Nusselt number are piled up to get a variety of graphs with their substantial interprtations by taking some standard values of different parameters implicated in the problem.

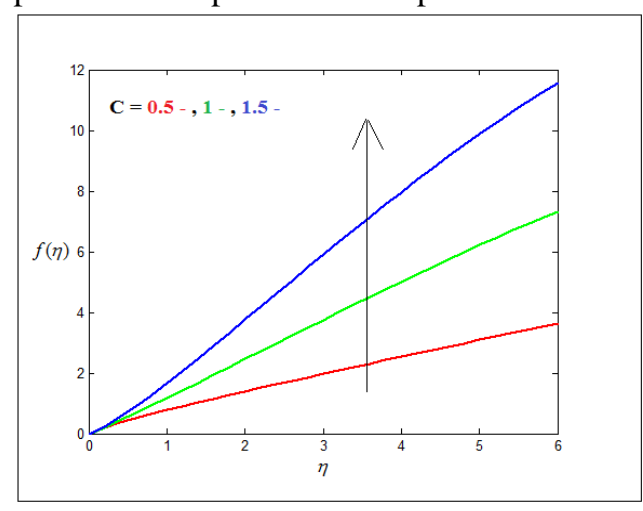

Figure 2: Velocity $f(\eta)$ versus $\eta$ under $M=3, P$ $=0.1$

Effect of parameter $C$ on velocity $f$

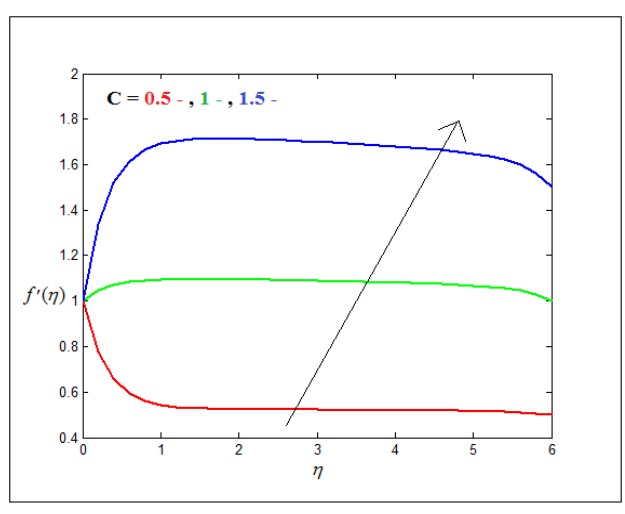

Figure 3: Velocity $\mathrm{f}^{\prime}(\eta)$ versus $\eta$ under, $M$ $=3, \mathrm{p}=0.1$ Effect of parameter $C$ on velocity $f^{\prime}$

Figures 2 and 3 demonstrate the impact of stretch parameter on the profile of the velocity. The $f$ and $\mathrm{f}^{\prime}$ velocity profiles areaccelerated for differetnt values of stretching parameter $(\mathrm{C}=0.5,1,1.5)$. Furthermore, both the figure shows the fact that the velocity distribution is directly proportional to the stretching parameter $\mathrm{C}$. 


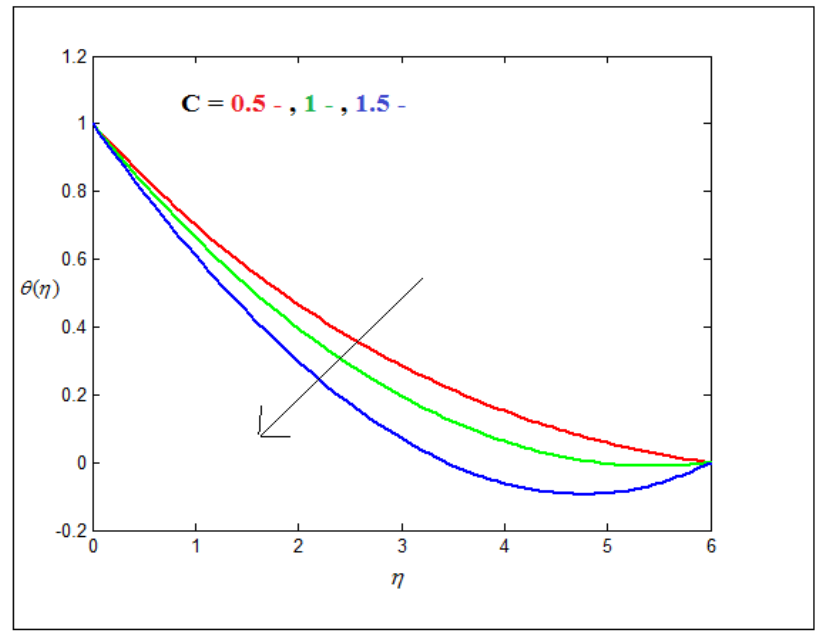

Figure 4: Température $\theta(\eta)$ versus $\eta$ Under $M$

$$
\begin{gathered}
=1, \operatorname{Pr}=0.7,|B|=0.1, \mathrm{P}=0.1 \\
\text { Effect of parameter } C \text { on } \theta
\end{gathered}
$$

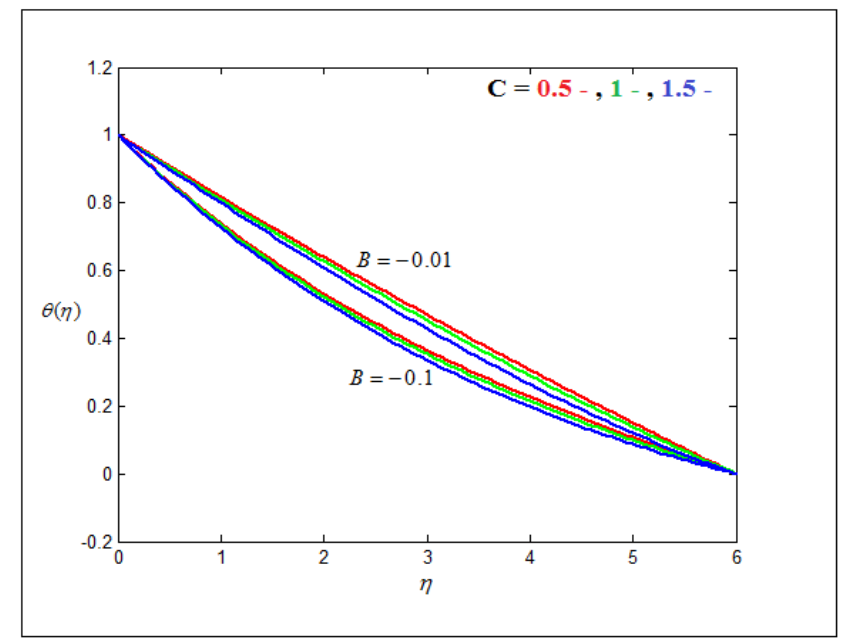

Figure 5: Température $\theta(\eta)$ versus $\eta$ Under

$$
\begin{gathered}
\mathrm{M}=0.5, \mathrm{Pr}=0.7,|B|=0.1, \mathrm{P}=0.1 \\
\text { Effect of parameter } C \text { and } \mathrm{B} \text { on } \theta
\end{gathered}
$$

Figures 4 depicts the consequence of stretching parameter on profile of temperature. It was noted that temperature profileis continuously reducedfor varying the stretching parameter $(\mathrm{C}=0.5,1,1.5)$ i.e. the temperature distribution is continuously moves down on account of stretching parameter.

Figure 5, shows the temperature profile for differentparameter values for heat generation and stretching parameter. It was observed that fluid temperature of the present flow problem is enlarged for boosting the heat generation which clearly satisfies the physical reality.

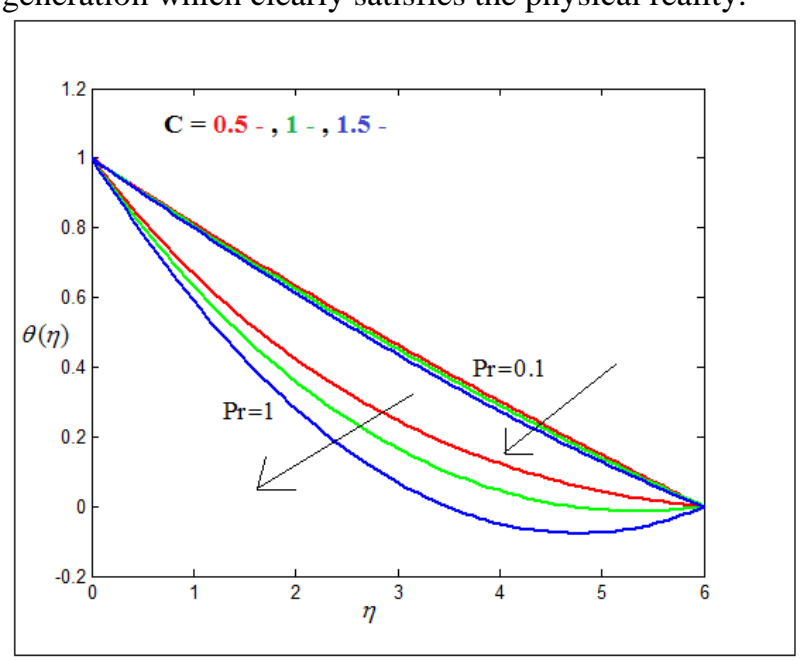

Figure 6: Temperature versus $\eta$ under $\mathrm{M}=1$, Comparison of resurts $9.1, \mathrm{P}=0.1$

Effects of parameter Pr and C on the profile of $\theta$

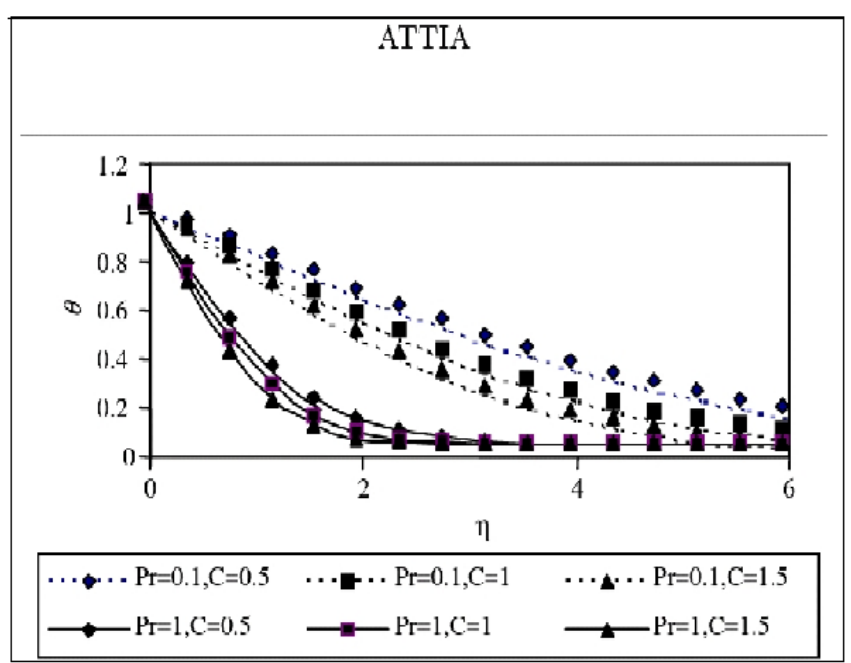

Figure 7 : (Fig 5 of H. A. Attia[9]): Temperature versus $\eta$ under $\mathrm{M}=1,|B|=0.1$

For comparing the results of the presenet research, the experiment of H. A. Attia [1] was considered. When figure 6 and 7 were compared (Figure 5 of the work done by H. A. Attia [1] ), we observed the same kind of behavior due to the effects of $\operatorname{Pr}$ and $\mathrm{C}$ on temperature profile for fixed $\mathrm{M}=1$ and $|B|=0.1$. i.e., there is a significant impact of Prandtl number on thickness of thermal boundary layer. Higher value of Pr lower is the thickness for consequent number of stretching parameter. Similarly, the fluid temperature falls for enhancing values of stretching parameter. Thus, an excellent agreement exists among the results achieved by $\mathrm{H}$. A. Attia [1] and the present authors. 


\section{Concluding remarks}

(a) The velocity distribution is directly proportional to the stretching parameter.

(b) The temperature distribution is continuously moves down on account of stretching parameter.

(c) The fluid temperature is enlarged for boosting the heat generation.

(d) High Prandtl number values condensed the thickness of thermal boundary layer for consequent values of stretching parameter.

\section{References}

Attia, H. A. (2006). Stagnation point flow towards a stretching surface through a porous medium with heat generation, Turkish J. Eng. Env. Sci. 30, 299-306.

Ariel, P. D. (2003). Generalized three-dimensional flow due to a stretching sheet, Z. Angew. Math. Mech. $83,12,844-852$.

Carragher, P., Crane, L. J. (1982) .Heat transfer on a continuous stretching sheet, ZAMM. 62, 564-577.

Chiam, T. C. (1994). Stagnation point flow towards a stretching plate, J. Phys. Soc. Jpn. 63, 2443-2444.

Crane, L. J. (1970). Flow past a stretching plate, ZAMP. 21, 645-647.

Dutta, B. K. et al (1985). Temperature field in flow over a stretching sheet with uniform heat flux, Int. Comm. Heat Mass Transfer. 12, 1, 89-94.

Gupta, P. S. and Gupta, A. S., (1977). Heat and mass transfer on a stretching sheet with suction or blowing, Can. J. Chem. Eng. 55, 6, 744-746.

He, J. H. (2009). An elementary introduction to the homotopy perturbation method, Computers \& Mathematics with Applications. 57, 3, 410-412.

Hiemenz, H., Die Grenzschicht (1911). an einem in den gleich formigen flussig eingetacuhten geraden krebzylinder Dingl, Polytech. J. 32, 321-342.

Jankhal, A. K. (2014). Homotopy Perturbation Method for MHD boundary layer flow with low pressure gradient, Journal of Applied Fluid Mechanics 7, 1, 177-185.

Kazem, S., Shaban, M. and Abbasbandy, S. (2011). Improved analytical solutions to a stagnation-point flow past a porous stretching sheet with heat generation. Journal of the Franklin Institute. 348, 2044-2058.

Mahapatra, T. R., Gupta A. S. (2002). Heat transfer in stagnation-point flow towards a stretching sheet, Heat Mass Transfer. 38, 6, 517-521.

Nazar, R., Amin, N., Filip, D. and Pop, I. (2004). Stagnation point flow of a micropolar fluid towards a stretching sheet, Int. J. of Non-linear Mech. 39, 1227-1235.

White, M. F. (1991). Viscous Fluid Flow, McGraw-Hill, New York.

$$
\begin{array}{lll}
M_{1}=C .(C+M) & M_{2}=-\operatorname{Pp} B & \\
M_{3}=\sqrt{M}+\sqrt{M_{2}} & M_{4}=\sqrt{M}-\sqrt{M_{2}} & M_{5}=\frac{M_{1}}{M}-1 \\
C_{1}=-C_{2}-C_{3} & C_{2}=C_{3}-\frac{M_{5}}{\sqrt{M}} & C_{3}=\frac{M_{5} e^{6 \sqrt{M}}-\frac{M_{1}}{M}+C}{\sqrt{M}\left(e^{6 \sqrt{M}}-e^{-6 \sqrt{M}}\right)} \\
C_{4}=\frac{1}{1-e^{12 \sqrt{M_{2}}}} & C_{5}=-C_{4} e^{12 \sqrt{M_{2}}} & \\
A_{2}=-2 C_{1} \frac{M_{1}}{M} & A_{1}=2 C_{2} C_{3} M-2 C_{2} C_{3}-C_{1}^{2} & A_{4}=C_{1} C_{2}(M-2) \\
A_{5}=C_{1} C_{3}(M-2) & A_{3}=-\frac{M_{1}^{2}}{M^{2}} & A_{7}=C_{3}^{2}(M-1) \\
A_{8}=C_{2} M_{1}\left(1-\frac{2}{M}\right) & A_{6}=C_{2}^{2}(M-1) & A_{10}=\frac{A_{2}}{2 M} \\
A_{11}=\frac{A_{3}}{3 M} & A_{9}=C_{3} M_{1}\left(1-\frac{2}{M}\right) & A_{13}=\frac{A_{4}}{2 M}
\end{array}
$$




$$
\begin{aligned}
& A_{14}=\frac{A_{5}}{2 M} \\
& A_{15}=\frac{A_{6}}{6 M \sqrt{M}} \\
& A_{16}=\frac{A_{7}}{6 M \sqrt{M}} \\
& A_{17}=\frac{A_{8}}{4 M} \\
& A_{18}=\frac{A_{9}}{4 M} \\
& A_{19}=\frac{3 A_{8}}{4 M \sqrt{M}} \\
& A_{20}=\frac{3 A_{9}}{4 M \sqrt{M}} \\
& E_{1}=A_{13}-A_{19} \\
& E_{2}=A_{14}+A_{20} \\
& E_{3}=A_{15}-A_{16} \\
& E_{4}=\frac{-A_{12}+2 \sqrt{M}\left(A_{15}+A_{16}\right)+\left(E_{1}+E_{2}\right)}{\sqrt{M}} \\
& E_{5}=-\frac{12 A_{10}+108 A_{11}+A_{12}}{\sqrt{M}}+2\left(A_{15} e^{12 \sqrt{M}}+A_{16} e^{-12 \sqrt{M}}\right)+6\left(E_{1} e^{6 \sqrt{M}}-E_{1} e^{-6 \sqrt{M}}\right) \\
& +\frac{1}{\sqrt{M}}\left(E_{1} e^{6 \sqrt{M}}+E_{2} e^{-6 \sqrt{M}}\right)-36\left(A_{17} e^{6 \sqrt{M}}-A_{18} e^{-6 \sqrt{M}}\right)-\frac{12}{\sqrt{M}}\left(A_{17} e^{6 \sqrt{M}}+A_{18} e^{-6 \sqrt{M}}\right) \\
& C_{6}=E_{3}-C_{7}-C_{8} \\
& C_{7}=\frac{E_{5}-E_{4} e^{-6 \sqrt{M}}}{e^{6 \sqrt{M}}-e^{-6 \sqrt{M}}} \quad C_{8}=\frac{E_{5}-E_{4} e^{6 \sqrt{M}}}{e^{6 \sqrt{M}}-e^{-6 \sqrt{M}}} \\
& A_{21}=\operatorname{Pr} \cdot C_{1} \cdot C_{4} \cdot \sqrt{M_{2}} \\
& A_{22}=\operatorname{Pr} \cdot C_{1} \cdot C_{5} \cdot \sqrt{M_{2}} \\
& A_{23}=\operatorname{Pr} \cdot C_{2} \cdot C_{4} \cdot \sqrt{M_{2}} \\
& A_{24}=\operatorname{Pr} \cdot C_{3} \cdot C_{5} \cdot \sqrt{M_{2}} \\
& A_{25}=\operatorname{Pr} \cdot C_{2} \cdot C_{5} \cdot \sqrt{M_{2}} \\
& A_{26}=\operatorname{Pr} . C_{3} \cdot C_{4} \cdot \sqrt{M_{2}} \\
& A_{27}=\operatorname{Pr} \cdot C_{4} \cdot \frac{M_{1} \sqrt{M_{2}}}{M} \\
& A_{28}=\operatorname{Pr} \cdot C_{5} \cdot \frac{M_{1} \sqrt{M_{2}}}{M} \\
& C_{9}=\frac{B_{2}-B_{1} e^{-6 \sqrt{M_{2}}}}{e^{6 \sqrt{M_{2}}}-e^{-6 \sqrt{M_{2}}}} \\
& C_{10}=\frac{B_{1} e^{6 \sqrt{M_{2}}}-B_{2}}{e^{6 \sqrt{M_{2}}}-e^{-6 \sqrt{M_{2}}}} \\
& B_{1}=A_{31}-A_{32}-A_{33}+A_{34} \\
& \begin{aligned}
B_{2}=- & 6\left(A_{39} e^{6 \sqrt{M_{2}}}+A_{40} e^{-6 \sqrt{M_{2}}}\right)+36\left(A_{35} e^{6 \sqrt{M_{2}}}-A_{36} e^{-6 \sqrt{1}}\right. \\
+ & \left(A_{31} e^{6 M_{3}}-A_{32} e^{-6 M_{3}}\right)-\left(A_{33} e^{6 M_{4}}-A_{34} e^{-6 M_{4}}\right)
\end{aligned} \\
& A_{30}=\frac{A_{22}}{2 \sqrt{M_{2}}} A_{31}=\frac{A_{23}}{M_{3}^{2}-M_{2}} \\
& A_{29}=\frac{A_{21}}{2 \sqrt{M_{2}}} \\
& A_{33}=\frac{A_{25}}{M_{4}^{2}-M_{2}} \\
& A_{34}=\frac{A_{26}}{M_{4}{ }^{2}-M_{2}} \\
& A_{35}=\frac{A_{27}}{4 \sqrt{M_{2}}} \\
& A_{36}=\frac{A_{28}}{4 \sqrt{M_{2}}} \\
& A_{37}=\frac{A_{27}}{4 M_{2}} \\
& A_{38}=\frac{A_{28}}{4 M_{2}} \\
& A_{39}=-A_{29}+A_{37} \\
& A_{32}=\frac{A_{24}}{M_{3}^{2}-M_{2}} \text {, } \\
& A_{40}=-A_{30}-A_{38}
\end{aligned}
$$

